\title{
THE ANALYSIS OF RELATIONSHIP BETWEEN LESION OF FUNCTIONAL SYSTEMS OF NERVOUS SYSTEM AND SEVERITY OF FATIGUE IN PATIENTS WITH MULTIPLE SCLEROSIS
}

\author{
Olena Mialovytska \\ Department of Neurology \\ O. O. Bogomolets National Medical University \\ 13 Taras Shevchenko blvd., Kyiv, Ukraine, 01601 \\ salyuk@i.ua \\ Yuliia Khyzhniak \\ Department of Neurology \\ O.O. Bogomolets National Medical University \\ 13 Taras Shevchenko blvd., Kyiv, Ukraine, 01601 \\ khyzhniakyulia@gmail.com
}

\begin{abstract}
Despite numerous investigations, fatigue remains one of the least studied manifestations of MS. However, significant prevalence of this symptom proves importance of its study.

The aim of the study was to define a prognostic assessment of fatigue development accompanying lesions of certain functional systems and degree of disability.

Material and methods. 96 patients, aged 15 to 58 years with a diagnosis of MS have been examined. MS patients were examined with the same protocol, using formalized scales. To detect fatigue and determine the degree of its severity was used Fatigue Severity Scale (FSS). The degree of disability in patients was assessed by Kurtzke EDSS (Expanded Disability Status Scale). Statistical methods for the data analysis were also used, including methods of descriptive statistics, probability assessment and prognostic significance of obtained data using odds ratio (OR).

Results. Fatigue was detected in $49.1 \%$ of patients with a degree of disability lower than 4 EDSS scores and in $90.4 \%$ of patients with disability higher than 4 EDSS scores $(\mathrm{p}=0.0027)$. The assessment of functional systems at the time of observation has statistically significant prognostic values according to the following parameters: pyramidal system lesion $(\mathrm{OR}=7.43(2.43-22.76))$, coordination disorders $(\mathrm{OR}=4.60(1.05-20.25))$, cranial nerve lesion $(\mathrm{OR}=4.33(1.40-13.39)),(\mathrm{p}<0.05)$. Lesion of the pyramidal and coordination systems at the onset of the disease may increase risk for detection of fatigue higher than 4 scores in the follow-up observation (lesion of pyramidal system $-\mathrm{OR}=1.62(0.5-5.25)$, coordination disorders $\mathrm{OR}=1.41(0.58-5.16)$ ).

Conclusion. The results of our investigation showed that symptom of fatigue is observed in almost $90 \%$ of MS patients. The study showed that fatigue in MS patients depends on the degree of disability and lesions of certain functional systems.

Keywords: multiple sclerosis, fatigue, degree of disability, main symptoms of nervous system lesion.
\end{abstract}

\section{Introduction}

Multiple sclerosis (MS) is a chronic, progressive, inflammatory, autoimmune, neurodegenerative disease of central nervous system, characterized by various levels of physical, emotional, cognitive, and social difficulties. Over the past decades, its incidence and prevalence in the world has increased. Today MS is leading in the structure of neurological pathology and affects about $0.05-0.1 \%$ of adult population [1-3].

Today in the world, there are about 3 million people with multiple sclerosis, and in Ukraine the number is approaching 20 thousand people. According to WHO, MS is the major cause of permanent disability of young people among all neurological diseases [4, 5].

Fatigue is one of the most complex neurological symptoms and its mechanism of development is one of least studied. Fatigue affects 53-92\% of patients with MS, often limits their social activity, impacts the quality of life, deepens their disability, worsens the course of other symptoms of the disease. Two out of three MS patients consider that fatigue is one of the three major disabling 
factors; many of them assess fatigue as a leading symptom yet at the onset of the disease [6, 7]. Fatigue can occur in all MS forms at any stage and is reported to impact employment in $41 \%$ and social functioning in $25 \%$ of MS patients [8].

Fatigue is characterized by a lack of energy, feelings of exhaustion that are unaided by sleep, and the perception that one is unable to perform mental and physical activities [9]. Although fatigue has been studied extensively, in part because it affects such a wide range of clinical populations, there has been no unifying framework within which to understand fatigue [10].

Fatigue is a multifactorial phenomenon in MS and is attributed to primary or secondary disease mechanisms [11]. Primary factors may include inflammation, demyelination, and destruction of axons in the central nervous system, the presence of immune markers, and neuroendocrine system disturbances. Secondary factors may include sleep problems, depression or other psychological variables, medications, and lack of exercise [12].

Owing to the paucity of information on the pathogenesis of fatigue and its bearing on an individual with MS, it is important to investigate the associations between high levels of fatigue and other MS-related impairments, functional performance, and community participation. The associations between fatigue and other MS-related impairments, activity, and participation limitations need to be further examined. The current literature is ambiguous regarding the relationship between fatigue and disability status [13-16] as well as fatigue and physical activity [17-19] therefore warranting further investigation. In addition, associations between fatigue and other MS-related impairments, such as dynamic balance, walking ability, lesion of main symptoms of nervous system are still unknown.

\section{Aim}

To define a prognostic assessment of fatigue development accompanying lesions of certain functional systems and degree of disability.

\section{Material and methods}

A total of 96 patients (34 male and 62 female) aged 15 to 58 years (mean age $38.45 \pm 1.04$ ) with a diagnosis of MS were examined. Among the examined patients, 83 showed the signs of fatigue ( $88 \%$ of the total number of patients that were included in the study).

MS was diagnosed based on conventional clinical neurological and paraclinical examination using McDonald W.I. et al. (2010) criteria.

Duration of the disease ranged from 11 months to 29 years. According to the EDSS, the degree of disability ranged $1.5-6.5$ scores, on average $-3.8 \pm 0.3$ scores.

Considering the type of the disease course, patients with relapsing-remitting type significantly prevailed - $89(93 \%)$ in comparison to patients with secondary-progressive MS - 5 (5\%) and primary progressive MS - 2 (2\%).

Among the examined patients with fatigue symptom, the stage of MS exacerbation was observed in 34 people (35\%), the stage of remission - in 62 people $(65 \%)$.

For prognostic assessment of fatigue development, the relative risk of detection of this symptom higher than 4 scores was defined, depending on the degree of disability according to the EDSS (risk assessment in terms of odds ratios - OR and 95\% CI). So statistically significant risk of fatigue was found at $\mathrm{EDSS}>4$ scores $(\mathrm{OR}=6.6(1.6-37.9)),(\mathrm{p}<0.05)$.

Fatigue was detected in $49.1 \%$ of patients with a degree of disability lower than 4 EDSS scores and in $90.4 \%$ of patients with disability higher than 4 EDSS scores $(p=0.0027)$.

MS patients were examined with the same protocol, using formalized scales. To detect fatigue and determine the degree of its severity, Fatigue Severity Scale - FSS (Krupp et al., 1989) was used. The degree of disability in patients was assessed by Kurtzke EDSS (Expanded Disability Status Scale).

Data analysis statistical methods were also used, including methods of descriptive statistics, probability assessment and prognostic significance of obtained data using odds ratio (OR). 


\section{Results}

Given the variety of complaints and neurological disorders in patients with MS, fluctuations of their severity and impairments in different functional systems of nervous system, the relationship between the initial symptoms of the disease and its clinical manifestations at the time of examination was observed. The onset of MS in the form of sensory impairment was found in 21 patients (25\%), optic nerve lesion - in 17 patients (21\%), pyramidal tract lesion - in 16 patients (19\%), impaired motor coordination - in 15 patients $(18 \%)$, cranial nerve disorders - in 10 patients $(12 \%)$, general weakness - in 4 patients (5\%) (Fig. 1).
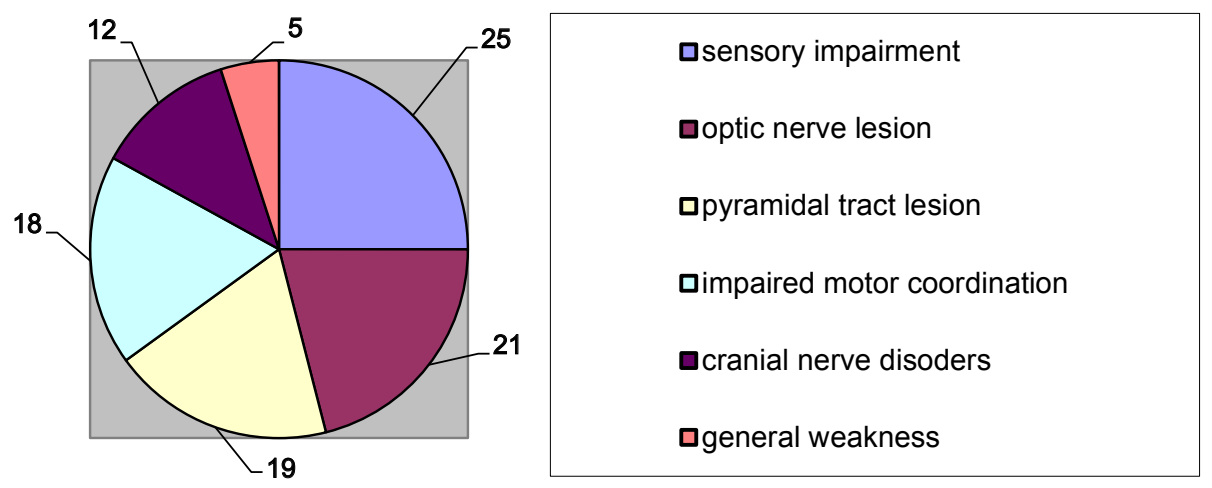

Fig. 1. Distribution of initial symptoms in MS patients with fatigue, in \%

At the time of examination, the following results were obtained: the main symptoms of pyramidal system lesion were found in 47 patients $(57 \%)$, predominant lesion of coordination system - in 14 patients $(17 \%)$, sensory impairment - in 11 patients $(13 \%)$, cranial nerve disorders - in 6 patients $(7 \%)$, optic nerve lesion - in 3 patients $(4 \%)$, lesion of pelvic organ function - in 2 patients (2\%) (Fig. 2).
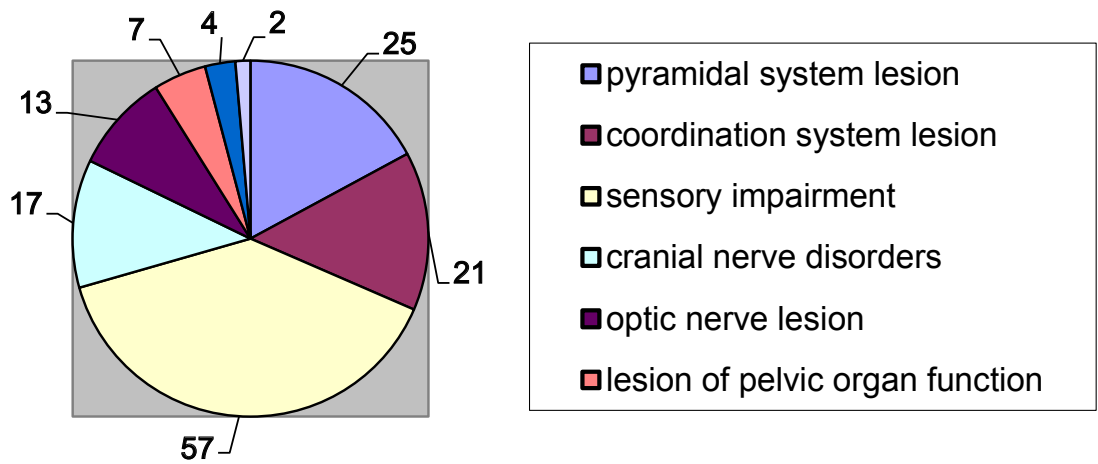

Fig. 2. Distribution of the main symptoms of nervous system lesion in MS patients with fatigue at the time of examination, in $\%$

For prognostic assessment of fatigue development, accompanied by impairment in different functional systems, the relative risk of detection of this symptom higher than 4 scores was defined, depending on presence or absence of functional system lesions (risk assessment in terms of odds ratios - OR and $95 \% \mathrm{CI}$ ).

Lesion of the pyramidal and coordination systems at the onset of the disease may increase the risk for detection of fatigue higher than 4 scores in the follow-up observation (lesion of pyramidal system $-\mathrm{OR}=1.62(0.5-5.25)$, coordination disorders $\mathrm{OR}=1.41(0.58-5.16))$. Generally, the whole range of initial manifestations does not result in statistically significant prognostic assessment for high rates of fatigue ( $\mathrm{p}>0.05)$ (Fig. 3). 
OR $(95 \% \mathrm{Cl})$

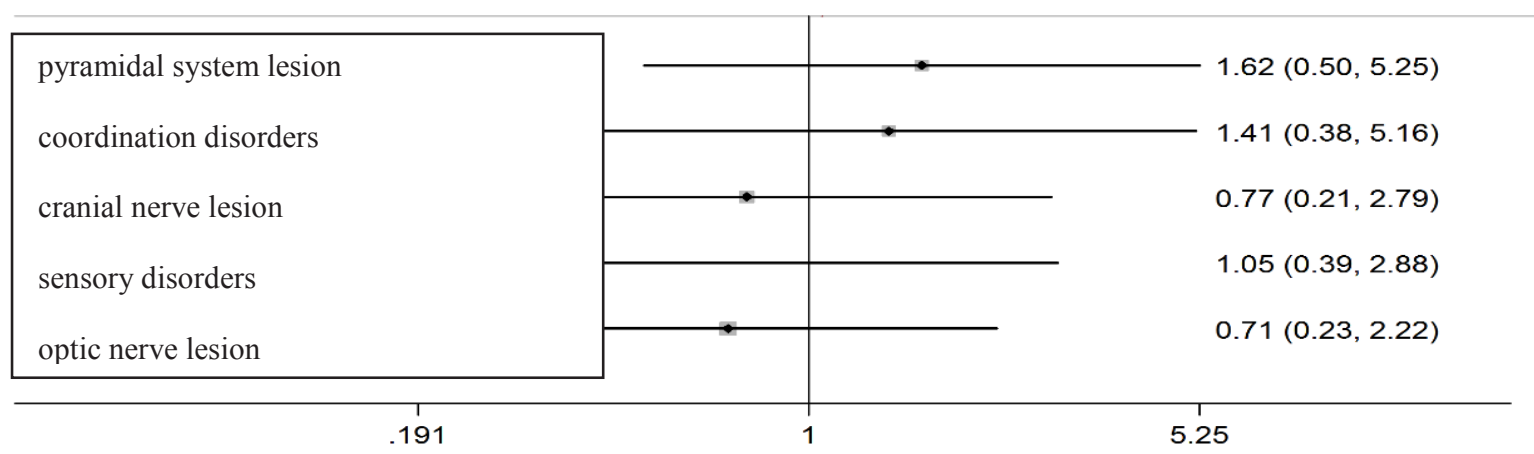

Fig. 3. Prognostic assessment of fatigue development higher than 4 FSS scores in case of lesion of particular functional systems at the onset of the disease (estimated odds ratio OR)

Assessment of functional systems at the time of observation has statistically significant prognostic values according to the following parameters: pyramidal system lesion $(\mathrm{OR}=7.43(2.43-22.76)$ ), coordination disorders $(\mathrm{OR}=4.60(1.05-20.25))$, cranial nerve lesion $(\mathrm{OR}=4.33(1.40-13.39)),(\mathrm{p}<0.05)$ (Fig. 4).

OR $(95 \% \mathrm{Cl})$

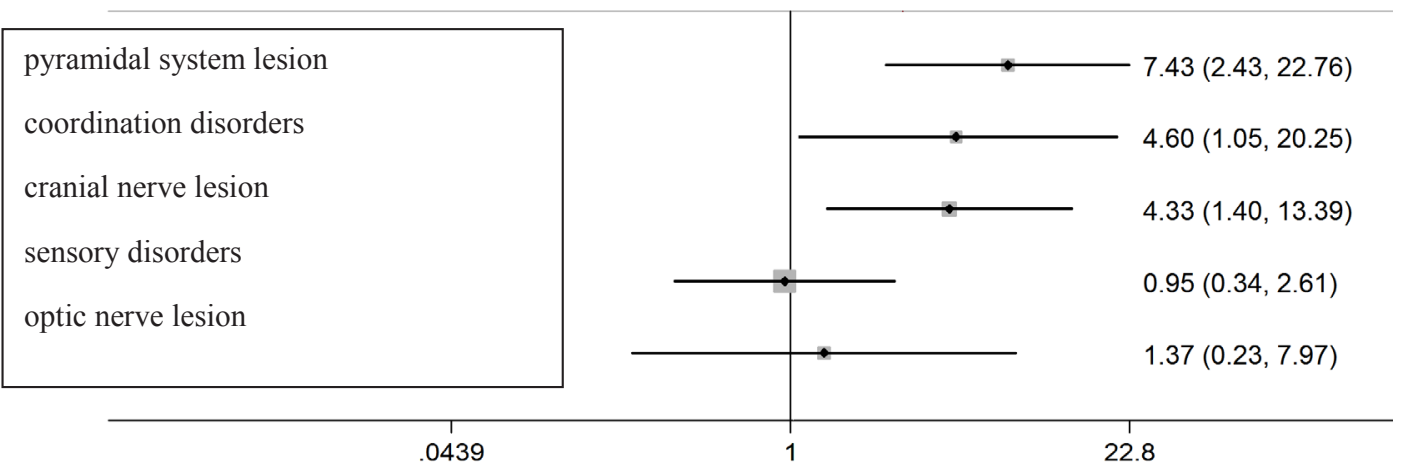

Fig. 4. Prognostic assessment of fatigue development higher than 4 FSS scores in case of lesion of particular functional systems at the moment of examination (estimated odds ratio OR)

The analysis of the relationship between lesion of functional systems and development of fatigue symptoms showed that lesions of pyramidal and coordination systems at the onset of the disease affect development of fatigue higher than 4 points during the follow-up observation. Lesions of pyramidal, coordination systems or cranial nerves at the time of examination had accurate prognostic assessment for the development of moderate and severe fatigue.

\section{Discussion}

The results of our investigation showed that the symptom of fatigue is observed in almost $90 \%$ of MS patients with different types of demyelinating process course and on different stages of the disease. This symptom occurs in patients of all ages, even at the very onset of the disease.

Our data also confirm the role of disability in fatigue development: $90.4 \%$ of respondents with a degree of disability of 4 points on the EDSS scale reported this symptom, unlike patients with mild disability among whom only $49.1 \%$ patients showed fatigue symptoms.

The analysis of the relationship between lesion of functional systems and development of fatigue symptoms showed that lesions of pyramidal and coordination systems at the onset of the disease affect development of fatigue higher than 4 points during the follow-up observation. Le- 
sions of pyramidal, coordination systems or cranial nerves at the time of examination had accurate prognostic assessment for the development of moderate and severe fatigue.

The results of our research coincide with ones of other investigators [19-23]. The major limitation of our work was retrospective character of the study, not allowing correct identification of prognostic factors.

Our future studies will be targeted at the prospective analysis of the relationship between lesions of certain functional systems and development of fatigue in patients with MS.

\section{Conclusions}

1. The results of our investigation showed that the symptom of fatigue is observed in almost $90 \%$ of MS patients.

2. Fatigue in MS patients depends on degree of disability on the EDSS scale.

3. The analysis of the relationship between lesion of functional systems and development of fatigue symptoms showed that lesions of pyramidal and coordination systems at the onset of the disease affect development of fatigue higher than 4 points during the follow-up observation.

4. Lesions of pyramidal, coordination systems or cranial nerves at the time of examination had accurate prognostic assessment for the development of moderate and severe fatigue.

\section{References}

[1] Johnson, S. L. (2008). The concept of fatigue in multiple sclerosis. The Journal of Neuroscience Nursing, 40 (2), 72-77. doi: 10.1097/01376517-200804000-00005

[2] Amato, M. P., Portaccio, E. (2012). Management options in multiple sclerosis-associated fatigue. Expert Opinion on Pharmacotherapy, 13 (2), 207-216. doi: 10.1517/14656566.2012.647767

[3] Cook, K. F., Bamer, A. M., Roddey, T. S., Kraft, G. H., Kim, J., Amtmann, D. (2013). Multiple Sclerosis and Fatigue. Physical Medicine and Rehabilitation Clinics of North America, 24 (4), 653-661. doi: 10.1016/j.pmr.2013.06.006

[4] Steens, A., de Vries, A., Hemmen, J., Heersema, T., Heerings, M., Maurits, N., Zijdewind, I. (2012). Fatigue Perceived by Multiple Sclerosis Patients Is Associated With Muscle Fatigue. Neurorehabilitation and Neural Repair, 26 (1), 48-57. doi: 10.1177/1545968311416991

[5] Induruwa, I., Constantinescu, C. S., Gran, B. (2012). Fatigue in multiple sclerosis - A brief review. Journal of the Neurological Sciences, 323 (1-2), 9-15. doi: 10.1016/j.jns.2012.08.007

[6] Kluger, B. M., Krupp, L. B., Enoka, R. M. (2013). Fatigue and fatigability in neurologic illnesses: Proposal for a unified taxonomy. Neurology, 80 (4), 409-416. doi: 10.1212/wnl.0b013e31827f07be

[7] Bakshi, R. (2003). Fatigue associated with multiple sclerosis: diagnosis, impact and management. Multiple Sclerosis Journal, 9 (3), 219-227. doi: 10.1191/1352458503ms904oa

[8] Thorsten, R., John, H., Nathaniel, B. (2016). Fatigue in Multiple sclerosis: misconceptions and future research directions. Frontiers in Neurology, 7, 22-28. doi: 10.3389/fneur.2016.00122

[9] Harrington, M. E. (2012). Neurobiological studies of fatigue. Progress in Neurobiology, 99 (2), 93-105. doi: 10.1016/j.pneurobio.2012.07.004

[10] Dobryakova, E., Helen, M., DeLuca, J., Glenn, R. (2015). The dopamine imbalance hypothesis of fatigue in multiple sclerosis and other neurological disorders. Frontiers in Neurology, 6, 35-39. doi: 10.3389/ fneur.2015.00052

[11] Kos, D., Kerckhofs, E., Nagels, G., D’hooghe, M. B., Ilsbroukx, S. (2007). Origin of Fatigue in Multiple Sclerosis: Review of the Literature. Neurorehabilitation and Neural Repair, 22 (1), 91-100. doi: 10.1177/1545968306298934

[12] Kaminska, M., Kimoff, R. J., Schwartzman, K., Trojan, D. A. (2011). Sleep disorders and fatigue in multiple sclerosis: evidence for association and interaction. Journal of the Neurological Sciences, 302, 7-13. doi: 10.1016/j.jns.2010.12.008

[13] Garg, H., Bush, S., Gappmaier, E. (2016). Associations Between Fatigue and Disability, Functional Mobility, Depression, and Quality of Life in People with Multiple Sclerosis. International Journal of MS Care, 18 (2), 71-77. doi: 10.7224/1537-2073.2015-013 
[14] Mills, R. J., Young, C. A. (2011). The relationship between fatigue and other clinical features of multiple sclerosis. Multiple Sclerosis Journal, 17 (5), 604-612. doi: 10.1177/1352458510392262

[15] Rietberg, M. B., van Wegen, E. E., Uitdehaag, B. M., Kwakkel, G. (2011). The association between perceived fatigue and actual level of physical activity in multiple sclerosis. Multiple Sclerosis Journal, 17 (10), 1231-1237. doi: 10.1177/1352458511407102

[16] Learmonth, Y. C., Dlugonski, D. D., Pilutti, L. A., Sandroff, B. M., Motl, R. W. (2013). The reliability, precision and clinically meaningful change of walking assessments in multiple sclerosis. Multiple Sclerosis Journal, 19 (13), 1784-1791. doi: 10.1177/1352458513483890

[17] Blikman, L. J., van Meeteren, J., Horemans, H. L., Kortenhorst, I. C., Beckerman, H., Stam, H. J., Bussmann, J. B. (2015). Is Physical Behavior Affected in Fatigued Persons With Multiple Sclerosis? Archives of Physical Medicine and Rehabilitation, 96 (1), 24-29. doi: 10.1016/j.apmr.2014.08.023

[18] Feys, P., Gijbels, D., Romberg, A., Santoyo, C., Gebara, B., de Noordhout, B. M. et. al. (2012). Effect of time of day on walking capacity and self-reported fatigue in persons with multiple sclerosis: a multi-center trial. Multiple Sclerosis Journal, 18 (3), 351-357. doi: 10.1177/1352458511419881

[19] Suh, Y., Motl, R. W., Mohr, D. C. (2010). Physical activity, disability, and mood in the early stage of multiple sclerosis. Disability and Health Journal, 3 (2), 93-98. doi: 10.1016/j.dhjo.2009.09.002

[20] Patejdl, R., Penner, I. K., Noack, T. K., Zettl, U. K. (2016). Multiple sclerosis and fatigue: A review on the contribution of inflammation and immune-mediated neurodegeneration. Autoimmunity Reviews, 15 (3), 210-220. doi: 10.1016/j.autrev.2015.11.005

[21] Karadayi, H., Arisoy, O., Altunrende, B., Boztas, M. H., Sercan, M. (2014). The relationship of cognitive impairment with neurological and psychiatric variables in multiple sclerosis patients. International Journal of Psychiatry in Clinical Practice, 18 (1), 45-51. doi: 10.3109/13651501.2013.845221

[22] Bakshi, R., Shaikh, Z. A., Miletich, R. S., Czarnecki, D., Dmochowski, J., Henschel, K. et. al. (2000). Fatigue in multiple sclerosis and its relationship to depression and neurologic disability. Multiple Sclerosis, 6 (3), 181-185. doi: 10.1177/135245850000600308

[23] Roelcke, U. (2009). Pathophysiology of fatigue in multiple sclerosis. Multiple sclerosis, 56, 171-176. doi: 10.4324/9780203212974_chapter_17 